\title{
Plant-Derived Polysaccharide Supplements Inhibit Dextran Sulfate Sodium-Induced Colitis in the Rat
}

\author{
Lee Koetzner · Gary Grover · Jamie Boulet • \\ Henry I. Jacoby
}

Received: 6 December 2008/ Accepted: 8 May 2009/Published online: 10 June 2009

(c) The Author(s) 2009. This article is published with open access at Springerlink.com

\begin{abstract}
Several plant-derived polysaccharides have been shown to have anti-inflammatory activity in animal models. Ambrotose complex and Advanced Ambrotose are dietary supplements that include aloe vera gel, arabinogalactan, fucoidan, and rice starch, all of which have shown such activity. This study was designed to evaluate these formulations against dextran sulfate sodium (DSS)-induced colitis in rats and to confirm their short-term safety after 14 days of daily dosing. Rats were dosed daily orally with vehicle, Ambrotose or Advanced Ambrotose. On day six groups of rats received tap water or $5 \%$ Dextran Sulfate sodium. Ambrotose and Advanced Ambrotose significantly lowered the disease scores and partially prevented the shortening of colon length. An increase in monocyte count was induced by dextran sulfate sodium and inhibited by Ambrotose and Advanced Ambrotose. There were no observable adverse effects after 14-day daily doses. The mechanism of action of the formulations against DSS-induced colitis may be related to its effect on monocyte count.
\end{abstract}

Keywords Plant derived polysaccharides .

Dextran sulfate sodium · Rat · Colitis .

Ambrotose · Aloe

L. Koetzner · G. Grover · J. Boulet

Eurofins Product Safety Laboratories, Dayton, NJ, USA

H. I. Jacoby $(\bowtie)$

Discovery Research Consultants, 4119 Ocean Avenue,

Brigantine, NJ 08203, USA

e-mail: hijacoby@earthlink.net

G. Grover

Department of Physiology and Biophysics, Robert Wood

Johnson Medical School, Piscataway, NJ, USA

\section{Introduction}

Several plant-derived polysaccharides have been shown to be effective in animal colitis models or IBD. Ambrotose complex (AMB) and Advanced Ambrotose powder (AdvAMB) are dietary supplement formulas that include these plant-derived polysaccharides. AdvAMB also contains galactofucan sulfate (fucoidan). Aloe [1, 2], arabinogalactan [3], fucoidan [4], rice starch [5] and glucosamine [6] have all been shown to have activity in animal colitis models. AMB and AdvAMB provide a good source of dietary fiber and immune system function [7] that can positively influence both gastrointestinal health and may have additional pharmacological activity due to the various polysaccharide components. Therefore it was of interest to evaluate the potential for AMB and AdvAMB for inhibitory activity in a rat model of colitis.

AMB can be digested by human colonic bacteria into lower molecular weight oligo- and monosaccharide and may then be absorbed through the intestinal mucosa [8]. The exact mechanism(s) of action of AMB products have not been established. Schley and Field [7] showed that dietary fibers and probiotics have immune-enhancing effects. Mannans and related complex carbohydrates have also been observed to stimulate immune response by activation of macrophages and stimulation of $\mathrm{T}$ cells [9]. The addition of soluble mannans reduces myeloperoxidasemediated killing in a dose dependent manner [10]. Studies have shown that probiotics administered to patients with inflammatory bowel disease have a positive effect [11].

Dextran Sulfate Sodium (DSS)-induced colitis has been shown to be a good model for inflammatory bowel disease (IBD). It produces significant colonic pathological changes in mice [12] and rats [13]. Studies have also shown that agents effective clinically are also effective in this model $[14,15]$. 
IBD includes both ulcerative colitis and Crohn's disease and comprises a family of diseases affecting both small and large intestines. The etiology is still unknown; however, several factors have been implicated in both the initiation and continuation of the pathology [16]. Current treatment consists of acute and maintenance dosing. Corticosteroids, aminosalicylate, immunomodulatory agents, antibiotics and anti-TNF $\gamma$ antibodies have all been used with limited success. Complementary and alternative therapy for IBD has been reviewed recently by Langmead and Rampton [17].

This study was designed to assess the possible protective effect of AMB and AdvAMB against DSS-induced colitis using prevention of colonic shortening and reduction of the Disease Activity Index. Terminal blood samples were also taken to evaluate any potential effects on blood cell count, clinical chemistry and lipid profile. A second arm of the study was designed to evaluate their short-term safety. $\mathrm{AMB}$ and AdvAMB were evaluated at doses comparable to, and ten times higher than, doses used in humans. Polysaccharide dosing prior to induction of DSS-induced colitis was used.

\section{Methods}

Selection of Animals One hundred healthy male SpragueDawley rats (Ace Animals, Boyertown, PA.) were weighed and assigned randomly to ten groups. The rats were acclimated to the housing facilities for 3 days prior to testing. The protocols were approved by the EPSL Institutional Animal Care and Use Committee.

Dosing All doses were administered daily on an $\mathrm{mg} / \mathrm{kg}$ basis. Each animal was dosed in the morning, starting on day 0 , by the oral route using a ball tipped needle attached to the appropriate size syringe. Vehicle (water), AMB, or AdvAMB suspended in water were administered at the appropriate dose to rats for 14 days.

Food Rats had access to Purina 5012 rat chow at all times. Housing Rats were housed singly in cages conforming to the latest NIH guide for the care and use of laboratory animals in a temperature-controlled room with a $12 \mathrm{~h}$ light/ dark cycle.

Dextran Presentation On day 6 of the study the rats were treated with the appropriate test article, the water supply was then removed and replaced by a water bottle containing either 5\% DSS (Dextran sulfate sodium: MW: 40,000 Lot\# DS40-011, Dextran Products Limited, Scarborough, Ontario Canada) dissolved in tap water or tap water. Approximately $70 \mathrm{ml} / \mathrm{rat} /$ day of fluid was provided from day 6 through the day of necropsy. No other source of fluids was available. Bottles were refilled daily with fresh DSS solution or tap water.
Bodyweight and Observation Rats were weighed on days 0 , $6,9,10,11,12,13$ and 14. The consistency of stools (formed, soft, mixed [soft and diarrhea]) and condition of the rats were evaluated at that time.

Blood Samples Blood samples $(\sim 3.5 \mathrm{ml})$ were taken by intraorbital bleeding under isofluorane anesthesia on days 0 and 14 (terminal). Samples were treated in the appropriate way depending on the analysis.

Blood Studies Aliquots of blood were prepared for the following analyses.

- Lipid profile (0.5 ml serum): Cholesterol, LDL, HDL and triglycerides; lipids were analyzed on an Olympus analyzer.

- Clinical chemistry: ALT (SGPT), AST (SGOT), GGT, $\mathrm{CK}$, total bilirubin, albumin, total protein, BUN, creatinine, glucose; $0.5 \mathrm{ml}$, EDTA blood. Hematology analyzed on Abbott Cell Dyn 3500.

- Blood cell count: $0.5 \mathrm{ml}$, EDTA blood and blood smear; blood cell count analyzed on Abbott Cell Dyn 3500; differential analyzed using Olympus AU2700.

The studies were performed at IDEXX Preclinical Services, North Grafton, MA.

Necropsy The study was terminated on day 14 after obtaining body weights and making observations. Rats were anesthetized with isoflurane. A midline incision was made and a stool sample was obtained from the rectum or colon and placed on a slide for testing for occult blood (Cacogenics Stool Blood Test, SB-21, Single Slide Professional Kit, Laboratory Diagnostics Co., Cacogenics, Morganville, NJ). Occult blood was determined by placing two drops of the reagent onto the stool sample on the slide and observing a change of color. The presence of occult blood was graded using a score of 0 , for no color change; 1 , for a very light blue color $( \pm)$ taking over 30 s to appear; 2, for a blue color developing in $30 \mathrm{~s}$ or more $(+)$; 3 , for an immediate change in color occurring in less than $30 \mathrm{~s}$ $(++)$; and 4, for gross blood observable on the slide. The colon was gently stretched and the distance from the colocecal junction to the end of the distal rectum was measured to the nearest $0.1 \mathrm{~cm}$. Five colons from each group were preserved in $10 \%$ buffered formalin and the other five flash frozen in a mixture of carbon dioxide and ethanol and preserved in $\mathrm{a}-70^{\circ} \mathrm{C}$ freezer in case histopathology or analysis of tissue cytokines were required.

\section{Statistical Analysis}

The scores for each parameter for each rat were added and then divided by three to give a DAI score for each rat. The scores for stool consistency and occult blood for each rat were added and then divided by two to give a DAINWT score for each rat (Table 1). Statistical comparisons were 
done using the non-parametric method of Kruskal-Wallis with Dunn's multiple comparison tests. The significance level was $P<0.05$.

For colon length and body weight, comparisons were done using one-way ANOVA and Bonferroni multiple comparisons test. The significance level was $P<0.05$.

Statistical Analysis of Clinical Chemistry, CBC,

Differentials, and Lipid Profile Assays

Data (terminal values only) from each individual experimental time point were analyzed by one-way analysis of variance (ANOVA). For each comparison, the $F$ ratio from the ANOVA was used to estimate the probability, or $P$ value, that the data were randomly distributed across groups. The main effect $P$ value represents the probability that the experimental groups had no effect on the variable being tested in that ANOVA. A $P$ value of $<0.05$ was used as the cutoff. Dennett's multiple comparison test was used for the post hoc comparisons. The low and high dose treatment groups were compared to the vehicle treated group and a significance level estimated for these comparisons. The same water group was used for both AMB and AdvAMB groups. Prism Version 5.01 (GraphPad Software, San Diego, CA) was used for all statistical analyses.

Table 1 Disease activity index (DAI) and DAI without weight parameter (DAINWT)

\begin{tabular}{llll}
\hline Score & Weight loss $\%$ & Stool consistency & Blood in feces \\
\hline 0 & 0 or gain & Normal & Negative \\
1 & $-1-4.9 \%$ & & Occult blood \pm \\
2 & $5.0-9.9 \%$ & Loose stools & Occult blood + \\
3 & $10-20 \%$ & & Occult blood ++ \\
4 & $>20 \%$ & Diarrhea & Gross blood \\
\hline
\end{tabular}

Test Agents

Ambrotose complex (US 37101, Lot \# NO7090154) and Advanced Ambrotose powder (US37601, Lot \# NO706 0313) were supplied by Mannatech, Inc. (Coppell, Texas, USA) (Table 2).

\section{Results}

Rats were sacrificed on the eighth day of access to DSS in tap water. Rats receiving oral vehicle and access to DSS showed signs of diarrhea and bloody stools indicating a moderate degree of colitis. Rats receiving vehicle, AMB or AdvAMB plus access to tap water showed no signs of changes in stool consistency or other signs of colitis.

\section{Disease Activity Index}

Rats receiving 5\% DSS as the sole source of liquid showed a significant increase in the Disease Activity Index with and without the weight loss parameter (DAI and DAINWT). Since there was no DSS-associated loss of body weight, weight loss was not a significant factor in determining the disease index. The DAINWT (DAI without weight parameter) is a more useful index of the degree of colitis when weight loss is not observed with DSS treatment. DSS produced a statistically significant increase in both DAI and DAINWT (Table 3). Rats receiving AMB and AdvAMB and exposed to 5\% DSS showed a doserelated trend to lower indices but a statistically significant inhibition was only noted with the high dose of AdvAMB $(574 \mathrm{mg} / \mathrm{kg}$ PO). The DAI and DAINWT scores of all groups of rats receiving daily administration of vehicle, AMB, AdvAMB and access only to tap water were similar. The administration of AMB and AdvAMB in rats allowed water instead of 5\% DSS did not induce any signs of

Table 2 Ambrotose and Advanced Ambrotose compositions averages

\begin{tabular}{|c|c|c|c|c|c|c|c|c|c|c|}
\hline \multirow[t]{2}{*}{ Material ingredients } & & \multicolumn{9}{|c|}{ Total monosaccharide content \% (wt/wt) } \\
\hline & & Fucose & Rhammnose & Arabinose & Galactose & Glucose & Xylose & Mannose & $\begin{array}{l}\text { Galacturonic } \\
\text { acid }\end{array}$ & $\begin{array}{l}\text { Glucuronic } \\
\text { acid }\end{array}$ \\
\hline Aloe vera gel extract & Aloe barbadensis & & & $<1$ & 1 & 2 & 2 & 12 & 5 & \\
\hline Arabinogalactan & Larix species & & & 8 & 50 & $<1$ & & & & \\
\hline Gum ghatti & $\begin{array}{l}\text { Anogeissus } \\
\quad \text { latifolia }\end{array}$ & & 2 & 34 & 14 & & & 1 & 6 & 3 \\
\hline Gum tragacanth & $\begin{array}{l}\text { Astragalus } \\
\text { gummifer }\end{array}$ & 4 & 1 & 17 & 5 & 6 & 10 & & 5 & 1 \\
\hline Rice starch & Oryza sativa & & & & & & & & & \\
\hline $\begin{array}{l}\text { Galactofucan } \\
\text { sulfate (fucoidan) }\end{array}$ & $\begin{array}{l}\text { Undaria } \\
\quad \text { pinnatifida }\end{array}$ & 18 & & & 15 & & & & & \\
\hline
\end{tabular}

${ }^{a}$ Present only in advance ambrotose 
Table 3 Effect of Ambrotose and Advanced Ambrotose on disease activity

\begin{tabular}{llll}
\hline Daily oral dose $(\mathrm{mg} / \mathrm{kg})$ & $N$ & Mean DAI \pm SEM & Mean DAINWT \pm SEM \\
\hline Vehicle + 5\% DSS & 10 & $1.8 \pm 0.14^{\mathrm{a}}$ & $2.75 \pm 0.21^{\mathrm{a}}$ \\
AMB 37.7 + 5\%DSS & 10 & $1.63 \pm 0.13$ & $2.45 \pm 0.20$ \\
AMB 377.1 + 5\%DSS & 10 & $1.37 \pm 0.14$ & $2.05 \pm 0.22$ \\
AdvAMB 57.4 + 5\%DSS & 10 & $1.53 \pm 0.15$ & $2.3 \pm 0.23$ \\
AdvAMB 574.3 + 5\% DSS & 10 & $1.20 \pm 0.10^{\mathrm{b}}$ & $1.8 \pm 0.15^{\mathrm{b}}$ \\
Vehicle + water & 10 & $0.33 \pm 0.0$ & $0.5 \pm 0.0$ \\
AMB 37.7 + water & 10 & $0.33 \pm 0.0$ & $0.5 \pm 0.0$ \\
AMB 377.1 + water & 10 & $0.33 \pm 0.0$ & $0.5 \pm 0.0$ \\
AdvAMB 57.4 + water & 10 & $0.36 \pm 0.03$ & $0.55 \pm 0.05$ \\
Adv AMB 574.3 + water & 10 & $0.40 \pm 0.07$ & $0.6 \pm 0.1$ \\
\hline
\end{tabular}

$D A I$ disease activity index, DAINWT disease activity index without weight parameter, $S E M$ standard error of the mean, $A M B$ ambrotose complex, $A d v A M B$ advanced ambrotose powder

${ }^{\text {a }}$ Comparison of vehicle + tap water to vehicle + DSS tests. $P<0.05$ Kruskal-Wallis test with Dunn's multiple comparison test

b Comparison of vehicle + DSS to treatments + DSS tests. $P<0.05$ Kruskal-Wallis test with Dunn's multiple comparison test

intestinal pathology, fecal consistency changes or occult fecal blood.

\section{Colonic Shortening}

DSS produced a statistically significant shortening of the length of the colon in vehicle plus DSS treated groups (Fig. 1). Statistically significant inhibition of DSS-induced colonic shortening was observed with the $377 \mathrm{mg} / \mathrm{kg}$ dose of AMB and both doses of AdvAMB ( 57.4 and $574 \mathrm{mg} / \mathrm{kg} \mathrm{PO}$ ).

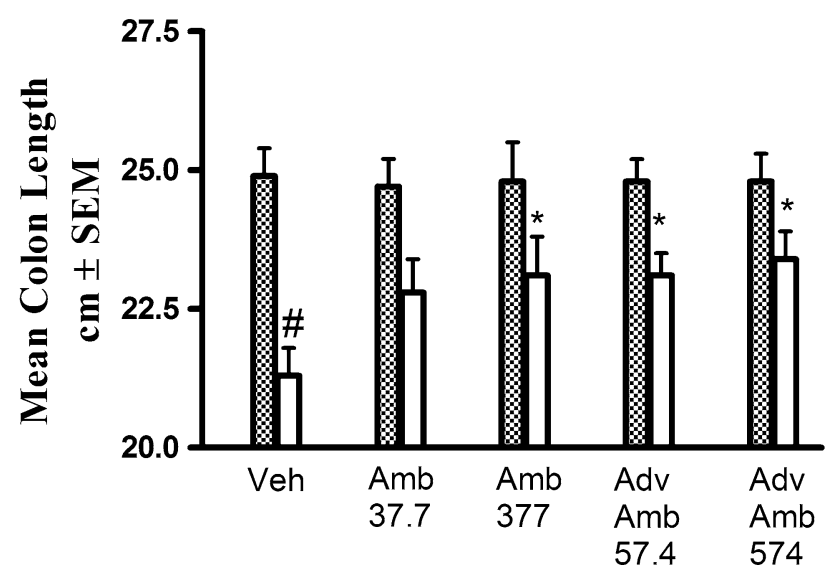

Dose mg/kg PO daily

Fig. 1 Colon length (mean, $\mathrm{cm} \pm$ SEM) at necropsy after 14 days of vehicle AMB or AdvAMB and oral dosing plus 8-day access to either DSS (clear bars) or tap water (dashed bars). Colon length was significantly shortened in the vehicle plus DSS-treated rats compared to the vehicle plus tap water group. AMB (377 mg/kg PO) and AdvAMB (57.4 and $574 \mathrm{mg} / \mathrm{kg} \mathrm{PO}$ ) significantly inhibited the DSSinduced colonic shortening. " Comparison of vehicle + tap water to vehicle + DSS. $P<0.05$ one-way ANOVA and Bonferroni multiple comparison test. * Comparison of vehicle + DSS to treatments + DSS. $P<0.05$ one-way ANOVA and Bonferroni multiple comparison test
AMB-inhibited DSS induced shortening of colon length by $47 \%$ and $53 \%$ with 37.7 and $377 \mathrm{mg} / \mathrm{kg} \mathrm{PO}$, respectively, while AdvAMB inhibited shortening 53\% and $61 \%$ at 57.4 and $574 \mathrm{mg} / \mathrm{kg}$ PO, respectively. Treatment with AMB or AdvAMB in rats with water access instead of 5\% DSS had no effect on colonic length compared to vehicle.

\section{Body Weight}

No statistically significant changes in body weights were noted in any of the groups receiving AMB or AdvAMB with or without exposure to 5\% DSS (Table 4).

\section{Blood Studies}

DSS treatment itself did not appear to affect hemoglobin or the absolute number of red blood cells, leukocytes, and lymphocytes (data not shown). However, exposure to 5\% DSS produced an increase in monocyte count. This increase was only statistically significant when monocyte counts were calculated as a percent in the vehicle plus tap water treatment group. AMB significantly decreased monocyte count in DSS-treated rats at $377 \mathrm{mg} / \mathrm{kg} \mathrm{PO}$, while AdvAMB significantly decreased the monocyte count in DSS-treated rats at both 57 and $577 \mathrm{mg} / \mathrm{kg}$ PO (Fig. 2). Neither AMB nor AdvAMB had any effect on these values in water drinking rats.

\section{Lipid Profile}

No statistically significant effects were noted due to treatment, DSS administration or treatment with low and high doses of AMB and AdvAMB on cholesterol, triglycerides, HDL and LDL levels (data not shown). 
Table 4 Change in mean body weight

\begin{tabular}{|c|c|c|c|c|c|c|c|c|}
\hline \multirow[t]{2}{*}{ Treatment mg/kg PO } & \multicolumn{8}{|c|}{ Mean body weight grams \pm SEM } \\
\hline & Day 0 & $\%$ & Day 12 & $\%$ & Day 13 & $\%$ & Day 14 & $\%$ \\
\hline Vehicle water + DSS & $345 \pm 4.7$ & $100 \pm 0$ & $400 \pm 5.4$ & $116 \pm 2.1$ & $403 \pm 5.0$ & $117 \pm 1.9$ & $403 \pm 5.0$ & $117 \pm 1.9$ \\
\hline AMB 37.7 + DSS & $349 \pm 5.4$ & $100 \pm 0$ & $403 \pm 6.0$ & $115 \pm 0.9$ & $406 \pm 5.4$ & $116 \pm 0.9$ & $402 \pm 3.8$ & $115 \pm 1.5$ \\
\hline AMB $377.1 \mathrm{~kg}+\mathrm{DSS}$ & $349 \pm 5.1$ & $100 \pm 0$ & $405 \pm 5.1$ & $116 \pm 1.8$ & $408 \pm 5.9$ & $117 \pm 2.2$ & $408 \pm 6.4$ & $117 \pm 2.0$ \\
\hline AdvAMB 57.4 + DSS & $348 \pm 5.1$ & $100 \pm 0$ & $392 \pm 6.6$ & $113 \pm 0.6$ & $396 \pm 7.1$ & $114 \pm 1.0$ & $399 \pm 7.4$ & $115 \pm 0.9$ \\
\hline AdvAMB $574.3+\%$ DSS & $344 \pm 5.1$ & $100 \pm 0$ & $393 \pm 6.1$ & $114 \pm 1.3$ & $398 \pm 5.7$ & $116 \pm 1.4$ & $397 \pm 7.4$ & $115 \pm 1.7$ \\
\hline Vehicle water + water & $347 \pm 2.9$ & $100 \pm 0$ & $404 \pm 5.3$ & $117 \pm 1.1$ & $405 \pm 6.8$ & $117 \pm 1.6$ & $405 \pm 6.8$ & $117 \pm 1.6$ \\
\hline AMB 37.7 + water & $348 \pm 4.7$ & $100 \pm 0$ & $409 \pm 9.4$ & $117 \pm 1.9$ & $413 \pm 9.4$ & $119 \pm 1.8$ & $414 \pm 9.1$ & $119 \pm 1.8$ \\
\hline AMB 377.1 + water & $349 \pm 4.5$ & $100 \pm 0$ & $403 \pm 6.9$ & $115 \pm 1.5$ & $404 \pm 7.2$ & $116 \pm 1.5$ & $406 \pm 7.5$ & $117 \pm 1.6$ \\
\hline AdvAMB $57.4+$ water & $346 \pm 4.3$ & 100. \pm 0 & $403 \pm 4.2$ & $117 \pm 2.0$ & $407 \pm 4.3$ & $118 \pm 1.9$ & $409 \pm 4.3$ & $118 \pm 1.7$ \\
\hline AdvAMB 574.3 + water & $348 \pm 5.5$ & $100 \pm 0$ & $408 \pm 10$ & $117 \pm 1.7$ & $412 \pm 11$ & $118 \pm 1.8$ & $417 \pm 11$ & $120 \pm 1.9$ \\
\hline
\end{tabular}

Change in rat body weight (mean \pm SEM) and mean \pm SEM percent of baseline weights. Percentage body weight was calculated using day 0 weight as the denominator

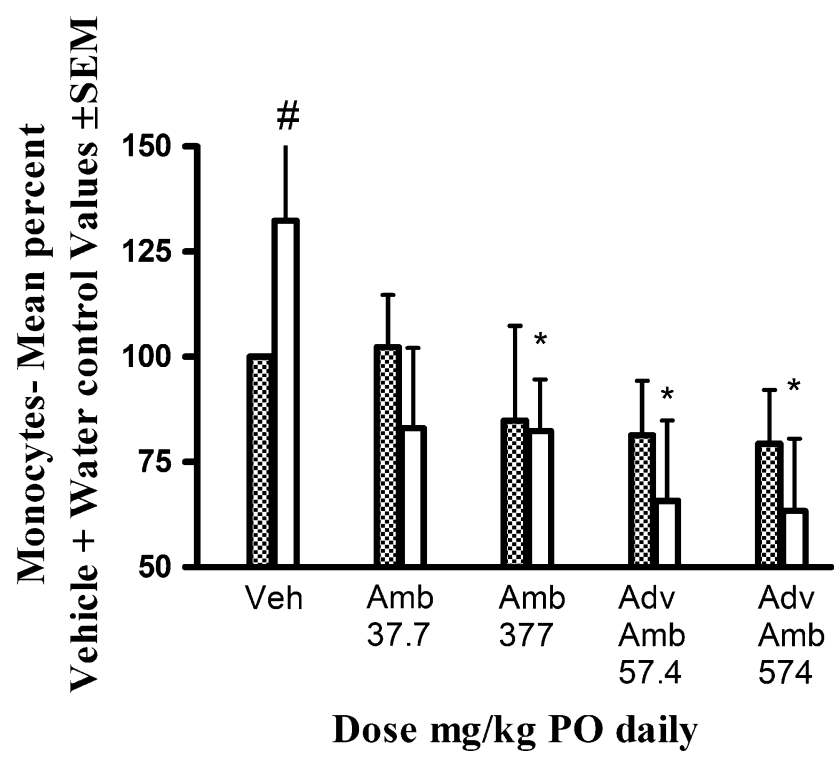

Fig. 2 Monocyte count calculated as a mean percent \pm SEM of the mean count in the vehicle plus tap water group at necropsy after 14 days of vehicle AMB or AdvAMB and oral dosing plus 8-day access to either DSS (clear bars) or water (dashed bars). Percent monocyte count was significantly increased in the vehicle plus DSS treated rats compared to the vehicle plus tap water group. AMB at $377 \mathrm{mg} / \mathrm{kg}$ PO and AdvAMB at 57.4 and $574 \mathrm{mg} / \mathrm{kg}$ PO daily significantly inhibited the mean percent of control monocyte count in DSS-induced treated rats. ${ }^{\#}$ Comparison of vehicle + tap water to vehicle + DSS. $P<0.05$ one-way ANOVA + Dunnett's test. * Comparison of vehicle + DSS to treatments + DSS. $P<0.05$ one-way ANOVA + Dunnett's test

\section{Blood Chemistry}

No statistically significant effects due to AMB or AdvAMB treatment plus DSS administration were observed on albumin, BUN, creatinine, glucose, ALT, AST, CK, and GGT (data not shown).

\section{Discussion}

Chronic daily oral dosing of AMB or AdvAMB dosed prior to and concomitantly with DSS significantly inhibited colitis produced by replacement of drinking water with a solution of 5\% DSS. AMB and AdvAMB lowered the incidence of diarrhea and occult blood in the feces as indicated by a decrease in the DAI. This was especially evident when the weight parameter was not used (DAINWT). The rats used in the study averaged between 300 and $350 \mathrm{~g}$ in order to provide sufficient blood for the analysis. The colitis produced was less severe than seen in smaller rats and mice. A longer exposure to DSS would likely have produced significant weight loss. There have been no published studies, to our knowledge, using rats of equivalent size in a DSS colitis model.

Shortening of the colon has been described in rats or mice receiving DSS $[12,14]$. The cause of this shortening has not been fully explained, but is a consistent finding in animal models of colitis. Using this criterion, both doses of AdvAMB and the high dose of AMB significantly inhibited the shortening in a dose-related manner. The percent inhibition was dose related and was greater than $47 \%$ for all doses. This inhibition is similar to or greater than that observed in studies with agents known to be effective clinically [15].

DSS was shown to produce a significant increase in monocyte count that was inhibited by AMB and AdvAMB. This effect was even more prominent if results were analyzed as a percent of mean monocyte count in the vehicle plus water group. The only significant changes in blood components in rats having access to either DSS or water and treatment with $\mathrm{AMB}$ or AdvAMB was a dose-related decrease in absolute monocyte counts. The effect on monocytes is especially interesting since Grip et al. [18] 
indicated that gut-resident and circulating monocytes are thought to be required for the initiation and maintenance of inflammatory bowel diseases.

The second portion of the study was designed to evaluate the safety of AMB and AdvAMB plus tap water when administered daily for 14 days. No significant changes in any of the parameters studied were observed. There were no treatment-related changes in body weight and no indication of any observation of gross pathology in the colon. Rats receiving low and high doses of $\mathrm{AMB}$ or AdvAMB and having access to tap water throughout the study showed no significant change in any of the disease indexes compared to vehicle, no indication of change in feces consistency or occult blood in the feces, nor any changes in colonic length. All control animals, as well as those dosed, had a minimal positive response in the occult blood test. This may have been due to the lab chow they were fed as undigested chow produces a positive result in the occult blood assay (data not shown). All blood values, clinical chemistry and cytokine analysis were within the control range.

Several of the components of AMB and AdvAMB have been shown to have effects on inflammatory diseases of the gastrointestinal tract either in animal models or on human disease. Langmead et al. [1] evaluated the gel from the aloe plant administered orally in a double-blind, randomized, placebo-controlled trial in the treatment of mild to moderate ulcerative colitis in 44 hospital out-patients given oral aloe vera gel (100 ml aloe vera gel) or placebo solution. Clinical remission, improvement and response occurred in nine of 30 patients given aloe vera, compared with one of 14 patients taking placebo. The Simple Clinical Colitis Activity Index and histological scores decreased significantly during treatment with aloe vera, but not with placebo. They concluded that oral aloe vera gel taken for 4 weeks produced a clinical response more often than placebo; it also reduced the histological disease activity and appeared to be safe. Langmead et al. [2] also showed a dose-dependent inhibitory effect on reactive oxygen metabolite production in an in vitro study using human colonic cells inhibiting the production of prostaglandin $\mathrm{E}_{2}$, but without effects on thromboxane $\mathrm{B}_{2}$ production. Aloe vera treatment (200 mg/kg PO) reduced leukocyte adherence and TNF $\gamma$ level, elevated IL-10 level and promoted gastric ulcer healing. Korkina et al. [19] evaluated the effects of a natural antioxidant preparation based on aloe vera and ubiquinone for potential activity against DSS-induced colonic inflammation and alterations of the intestinal electrophysiological activity and motility. They used 5\% dextran sulfate for 3 days, followed by $1 \%$ DSS for 4 days administered in drinking water. Lipoperoxidation, superoxide production, glutathione peroxidase, glutathione-S-transferase activities, and reduced glutathione content increased, and superoxide dismutase and catalase activities were sharply suppressed in colon tissue. Inflammation, electrical/mechanical impairment in the gut, and most of the oxidative stress parameters were improved substantially by pre-treatment, but not by simultaneous or post treatment.

Arabinogalactan has been reported to have positive effects on fecal chemistry [16]. Arabinogalactan is a nondigestible soluble dietary fiber that resists hydrolytic enzyme action and enters the large bowel intact where it is fermented by resident microflora. The effect of 15 and $30 \mathrm{~g}$ orally per day of a commercially available arabinogalactan from Western Larch produced significant increases in total fecal anaerobes. A significant increase in Lactobacillus spp. was observed when subjects consumed arabinogalactan for a total of 6 weeks with no significant changes in other microflora, fecal enzyme activity, transit time, frequency, fecal weight, fecal $\mathrm{pH}$ and short-chain fatty acids.

Matsumoto et al. [20] showed that fucoidans derived from Cladosiphon okamuranus Tokida ameliorates chronic colitis in the rat through the down-regulation of interleukin-6 production on colonic epithelial cells. Previous studies showed the interleukin (IL-6 STAT-3) signal was up-regulated in inflammatory bowel disease in both humans and animal models [21]. Fucoidan treatment caused a decrease in the DAI, and myeloperoxidase activity decreased in mice fed Cladosiphon fucoidan, but not Fucus fucoidan. The levels of IL-6 mRNA in colonic epithelial cells were lower in colitis-induced Balb/c mice fed Cladosiphon fucoidan than those fed a standard diet. Fucoidan improves mouse chronic colitis by down regulating the synthesis of IL-6 in the colonic epithelial cells. The authors concluded that fucoidan derived from C. Tokida might be useful as a dietary substance for patients with inflammatory bowel disease.

Rice starch type 3 (115 g/kg) was shown to block DSSinduced colonic shortening in rats after 7 days of dosing while fructo-oligosaccharides did not [22]. Rice starch is a substrate yielding high levels of butyrate [5]. Butyrate is recognized to be effective in patients with Crohn's disease and ulcerative colitis when administered by enema [23]; however, the effect is not present when given orally [24]. Sprague-Dawley rats received water or DSS and cecocolonic inflammatory injuries assessed macroscopically and histologically. At days 7 and 14, cecal and distal macroscopic and histological observations were improved in rice starch plus DSS and the butyrate levels were higher in the cecum of the rice starch plus DSS rats.

Glucosamine has been observed to decrease proinflammatory cytokine-induced ICAM-1 production in human conjunctival cells in vitro [25] and inhibit TNF $\alpha$ and IFN $\gamma$ induced production of ICAM 1 in human retinal cells in vitro [26]. Although these studies were not done in gastrointestinal tissue, ICAM 1 has been implicated in production of DSS-induced colitis in mice [15]. Glucosamine 
has also been shown to protect against ibuprofen-induced gastric lesions in rats [6], significantly prevented ibuprofen-induced depletion of protein and glycoprotein components and maintained the activities of membrane-bound ATPases as compared to the untreated ulcer induced group of rats. The authors ascribe the effectiveness of glucosamine in part to strengthening the mucosal barrier by increasing mucosal glycoprotein synthesis and to its free radical scavenging property.

AMB and AdvAMB both were effective in interfering with the pathological effects of 5\% DSS-induced colitis in the rat. Although the colitis was not severe, shortening of the colon, the presence of occult blood in the feces and either a change in consistency of the fecal pellets or diarrhea were noted. All these were attenuated by daily dosing of $\mathrm{AMB}$ and AdvAMB. The low doses tested were equivalent to the usual human dose.

Polysaccharides found in aloe vera have been reported to have antiviral, antibacterial, antifungal and antitumor activity [27]. This and the multiple effects of the other components of AMB and AdvAMB on butyrate, ICAM-1, $\mathrm{TNF} \gamma$, etc. may be additive and have a positive effect on the pathology and symptoms of IBD. A combination of these plant-derived materials may be more effective than any one administered alone. However, this conclusion would require additional testing of the individual components.

Daily oral dosing with AMB and AdvAMB were both shown to have significant effects in inhibiting DSS-induced colitis in rats at doses that are safe, even when administered at multiples of the human dose for 14 days. Administration of either of these formulations may be useful in improving the quality of life in IBD patients.

Acknowledgments We would like to thank Mannatech, Inc. for providing the test material and for funding.

Open Access This article is distributed under the terms of the Creative Commons Attribution Noncommercial License which permits any noncommercial use, distribution, and reproduction in any medium, provided the original author(s) and source are credited.

\section{References}

1. Langmead L, Feakins RM, Goldthorpe S, et al. Randomized, double-blind, placebo-controlled trial of oral aloe vera gel for active ulcerative colitis. Aliment Pharmacol Ther. 2004;19:739747. doi:10.1111/j.1365-2036.2004.01902.x.

2. Langmead L, Makins RJ, Rampton DS. Anti-inflammatory effects of aloe vera gel in human colorectal mucosa in vitro. Aliment Pharmacol Ther. 2004;19:521-527. doi:10.1111/j.13652036.2004.01874.x.

3. Robinson RR, Feirtag J, Slavin JL. Effects of dietary arabinogalactan on gastrointestinal and blood parameters in healthy human subjects. J Am Coll Nutr. 2001;20:279-285.
4. Patankar MS, Oehninger S, Barnett T, Williams RL, Clark GF. A revised structure for fucoid may explain some of its biological activities. J Biol Chem. 1993;268:21770-21776.

5. Moreau NM, Champ MM, Goupry SM, et al. Resistant starch modulates in vivo colonic butyrate uptake and its oxidation in rats with dextran sulfate sodium-induced colitis. J Nutr. 2004;134: 493-500.

6. Santhosh S, Anandan R, Sini TK, Mathew PT. Protective effect of glucosamine against ibuprofen-induced peptic ulcer in rats. J Gastroenterol Hepatol. 2007;22:949-953. doi:10.1111/j.14401746.2007.04840.x.

7. Schley PD, Field CJ. The immune-enhancing effects of dietary fibres and probiotics. Br J Nutr. 2002;87:S221-S230.

8. Sinnott RA, Ramberg J, Kirchner JM, et al. Utilization of arabinogalactan, aloe vera gel polysaccharides, and a mixed saccharide dietary supplement by human colonic bacteria in vitro. Int $J$ Probiotics Prebiotics. 2007;2:97-104.

9. Tizard IR, Carpenter RH, McAnalley BH, Kemp MC. The biological activities of mannans and related complex carbohydrates. Mol Biother. 1989;1:290-296.

10. Lehrer RI, Kline MJ. Leukocyte myeloperoxidase deficiency and disseminated candidiasis: The role of myeloperoxidase in resistance to Candida infection. J Clin Invest. 1969;48:1478-1488. doi:10.1172/JCI106114.

11. Guarner F. Probiotics in inflammatory bowel diseases. Br J Nutr. 2007;98:S85-S89. doi:10.1017/S0007114507832958.

12. Okayasu I, Hatakemaya S, Yamada M, Ohkusa T, Inagaki Y, Nakaya R. Novel method in the induction of reliable experimental acute and chronic ulcerative colitis in mice. Gastroenterology. 1990;98:694-702.

13. Stucchi AF, Shebani KO, Leeman SE, et al. NK-1 antagonist reduces colonic inflammation and oxidative stress in dextran sulfate-induced colitis in rats. Am J Physiol Gastrointest Liver Physiol. 2000;279:G1298-G1306.

14. Murthy SNS, Cooper HS, Shim H, Shah S, Ibrahim SA, Sedergran MT. Treatment of dextran sulfate sodium-induced murine colitis by intracolonic cyclosporin. Dig Dis Sci. 1993;38:17221734. doi:10.1007/BF01303184.

15. Bennett CF, Kombrust D, Henry S, et al. An ICAM-1 antisense oligonucleotide prevents and reverses dextran sulfate sodiuminduced colitis in mice. J Pharmacol Exp Ther. 1997;280:9881000.

16. Robinson M. Medical therapy of inflammatory bowel disease for the 21st century. Eur J Surg. 1998;582:90-98. doi:10.1080/ 11024159850191517.

17. Langmead L, Rampton DS. Review article: complementary and alternative therapies for inflammatory bowel disease. Aliment Pharmacol Ther. 2006;23:341-349. doi:10.1111/j.1365-2036. 2006.02761.x.

18. Grip O, Janciauskiene S, Lindgren S. Macrophages in inflammatory bowel disease. Curr Drug Targets Inflame Allergy. 2003;2:155-160. doi:10.2174/1568010033484179.

19. Korkina L, Suprun M, Petrova A, Mikhal'chik E, Luci A, De Luca C. The protective and healing effects of a natural antioxidant formulation based on ubiquinol and aloe vera against dextran sulfate-induced ulcerative colitis in rats. Biofactors. 2003;18:255-264. doi:10.1002/biof.5520180228.

20. Matsumoto S, Nagaoka M, Hara T, Kimura-Takagi I, Mistuyama K, Ueyama S. Fucoidan derived from Cladosiphon okamuranus Tokida ameliorates murine chronic colitis through the downregulation of interleukin-6 production on colonic epithelial cells. Clin Exp Immunol. 2004;136:432-439. doi:10.1111/j.1365-2249. 2004.02462.x.

21. Suzuki A, Hanada T, Mitsuyama K, et al. CIS3/SOCS3/SSI3 plays a negative regulatory role in STAT3 activation and 
intestinal inflammation. $J$ Exp Med. 2001;193:471-481. doi: 10.1084/jem.193.4.471.

22. Moreau NM, Martin LJ, Toquet CS, et al. Restoration of the integrity of rat caeco-colonic mucosa by resistant starch, but not by fructo-oligosaccharides, in dextran sulfate sodium-induced experimental colitis. Br J Nutr. 2003;90:75-85. doi:10.1079/BJN 2003867.

23. Breuer RI, Buto SK, Christ ML, et al. Rectal irrigation with shortchain fatty acids for distal ulcerative colitis. Preliminary report. Dig Dis Sci. 1991;36:185-187. doi:10.1007/BF01300754.

24. Kanauchi O, Iwanaga T, Mitsuyama K, et al. Butyrate from bacterial fermentation of germinated barley foodstuff preserves intestinal barrier function in experimental colitis in the rat model.
J Gastroenterol Hepatol. 1999;14:880-888. doi:10.1046/j.14401746.1999.01971.x.

25. Chen JT, Chen CH, Horng CT, et al. Glucosamine sulfate inhibits proinflammatory cytokine-induced ICAM-1 production in human conjunctival cells in vitro. J Ocul Pharmacol Ther. 2006;22:402416. doi:10.1089/jop.2006.22.402.

26. Chen JT, Liang JB, Chou CL, et al. Glucosamine sulfate inhibits TNF-alpha and IFN-gamma-induced production of ICAM-1 in human retinal pigment epithelial cells in vitro. Invest Ophthalmol Vis Sci. 2006;47:664-672. doi:10.1167/iovs.05-1008.

27. Leung MYK, Liu C, Koon JCM, Fung KP. Polysaccharide biological response modifiers. Immunol Lett. 2006;105:101-114. doi:10.1016/j.imlet.2006.01.009. 